\title{
PREVALÊNCIA DE INDICADORES DE RISCO PARA SURDEZ EM NEONATOS EM UMA MATERNIDADE PAULISTA
}

\author{
Prevalence of deafness risk indicators in newborns \\ in a São Paulo' upcountry materninty hospital
}

\author{
Bárbara Carolina Teixeira Amado ${ }^{(1)}$, Elizabeth Oliveira Crepaldi de Almeida ${ }^{(2)}$, Paloma Savioli Berni ${ }^{(3)}$
}

\begin{abstract}
RESUMO
Objetivo: identificar os indicadores de risco para perda auditiva em recém-nascidos de um hospital da rede pública de Campinas - São Paulo, no período de julho de 2007 a janeiro de 2008. Métodos: a amostra foi composta por 589 protocolos de neonatos avaliados no Programa de Triagem Auditiva Neonatal Universal, no período. Resultados: do total de protocolos estudados, 152 (25,8\%) evidenciaram presença de indicadores de risco para surdez em neonatos. Dentre eles, os mais prevalentes, em ordem decrescente, foram: antecedentes familiares (26,3\%), boletim Apgar de 0 a 4 no primeiro minuto $(23,6 \%)$, uso de medicamentos ototóxicos $(23,6 \%)$, permanência em incubadora $(22,3 \%)$, hiperbilirrubinemia $(21,7 \%)$ e permanência em Unidade de Terapia Intensiva Neonatal $(18,4 \%)$. Conclusão: o número de indicadores de risco encontrados por neonato variou de um a seis. Dentre os neonatos com indicadores de risco, apenas um bebê falhou nas três avaliações realizadas na triagem auditiva, sendo que, de acordo com seu protocolo, ele apresentava três indicadores de risco para surdez.
\end{abstract}

DESCRITORES: Audiologia; Indicador de Risco; Triagem

\section{INTRODUÇÃO}

A prevalência das perdas auditivas na infância é estimada em 1,5/1000, sendo que, entre as crianças com déficit permanente de audição, cerca de $90 \%$ apresentam distúrbios neurossensoriais, 5\% de condução e $5 \%$ mistos ${ }^{1-4}$. A meta primordial dos profissionais que trabalham com deficientes auditivos é a prevenção ${ }^{5}$. O diagnóstico audiológico e a intervenção precoce permitem que a criança com

(1) Aluna do Curso de Graduação em Fonoaudiologia da Pontifícia Universidade Católica de Campinas, PUCCAMP, Campinas, SP; Bolsista de Iniciação Científica; Membro do Grupo de Estudo dos Aspectos Moleculares da Perda Auditiva da Universidade Estadual de Campinas.

(2) Fonoaudióloga; Docente Titular do Curso de Fonoaudiologia da Pontifícia Universidade Católica de Campinas, PUCCAMP, Campinas, SP; Pesquisadora do Grupo de Estudo dos Aspectos Moleculares da Perda Auditiva da Universidade Estadual de Campinas; Doutora em Educação.

(3) Aluna do Curso de Graduação em Fonoaudiologia da Pontifícia Universidade Católica de Campinas, PUCCAMP, Campinas, SP; Bolsista de Iniciação Científica; Membro do Grupo de Estudo dos Aspectos Moleculares da Perda Auditiva da Universidade Estadual de Campinas. perda auditiva tenha desenvolvimento global semeIhante ao de crianças ouvintes ${ }^{6}$. As recomendações internacionais e nacionais, atualmente, propõem a identificação de perdas auditivas até os três meses de idade por meio da Triagem Auditiva Neonatal Universal (TANU) ${ }^{6-8}$.

Quanto mais cedo for a detecção da perda auditiva, mais precocemente medidas de intervenção poderão ser tomadas ${ }^{9}$, bem como a identificação da etiologia propicia maior controle e prevenção ${ }^{10}$. O desenvolvimento auditivo, que se inicia já na vida intra-uterina, pode ter problemas em decorrência da presença ou mesmo na ausência dos Indicadores de Risco para Deficiência Auditiva ${ }^{11}$. A qualidade de vida dos recém-nascidos de risco se tornou uma preocupação, exigindo-se a intervenção precoce, devido à probabilidade de sequelas neurológicas, déficits somáticos, dificuldades de aprendizagem, distúrbios de comportamento, anormalidades neuromotoras e de deficiência auditiva ${ }^{12}$.

São indicadores de risco para surdez em neonatos 7,8 : história familiar de deficiência auditiva congênita; infecção congênita (sífilis, toxoplasmose, rubéola, citomegalovírus, herpes); anomalias crânio-faciais (malformações de pavilhão auricular, 
meato acústico externo, ausência de filtrum nasal, implantação baixa da raiz do cabelo); peso ao nascimento inferior a $1500 \mathrm{~g}$; hiperbilirrubinemia (níveis séricos indicativos de exsanguíneo-transfusão); medicação ototóxica por mais de cinco dias (aminoglicosídeos ou outros, associados ou não aos diuréticos de alça); meningite bacteriana; boletim Apgar de 0 a 4 no $1^{\circ}$ minuto ou 0 a 6 no $5^{\circ}$ minuto; ventilação mecânica por período mínimo de cinco dias; sinais ou síndromes associadas à deficiência auditiva condutiva ou neurossensorial.

Avanços tecnológicos possibilitaram a substituição da triagem auditiva comportamental pela eletrofisiológica ${ }^{13}$. Duas técnicas fisiológicas são utilizadas: o potencial auditivo de tronco encefálico (PAETE) e o exame de emissões otoacústicas evocadas (EOAE), esta última sendo uma técnica eletroacústica. O programa de identificação precoce da deficiência auditiva deve contemplar a triagem auditiva por uma medida eletrofisiológica, a pesquisa dos indicadores de risco e o acompanhamento de todos os lactentes que apresentam tais indicadores ${ }^{14,15}$, além da investigação da etiologia. Em países desenvolvidos, estima-se que cerca de $60 \%$ dos casos de perdas auditivas pré-linguais apresentam bases genéticas ${ }^{16-18}$.

Por meio das Emissões Otoacústicas Evocadas, pode-se avaliar a função coclear de forma objetiva e em pequenas frações, desde a espira basal até a espira apical, pela variação das frequências dos estímulos ${ }^{19,20}$. As mais utilizadas são as Evocadas Transientes e as Produtos de Distorção, sendo que as Evocadas Transientes são as mais recomendadas para a Triagem Auditiva Neonatal - TAN ${ }^{21}$.

Também se deve investigar os fatores de risco, a observação do comportamento auditivo e a pesquisa do reflexo cócleo-palpebral ${ }^{22}$. Um estudo com 798 bebês indicou que 54\% apresentavam pelo menos um indicador de risco; dentre aqueles do alojamento conjunto, o indicador de risco prevalente foi o antecedente familiar de perda auditiva e entre os bebês internados, o uso de medicamentos ototóxicos ${ }^{23}$. Alguns recém-nascidos com indicadores de risco podem passar na triagem auditiva neonatal, mas necessitam de monitoramento periódico a cada seis meses até a idade de três anos, pois possuem risco para o aparecimento tardio de perda auditiva condutiva ou neurossensorial ${ }^{14}$.

O presente estudo visa determinar a prevalência de indicadores de risco em neonatos avaliados por meio do exame de Emissões Otoacústicas Evocadas Transientes - EOAET em uma maternidade situada em Campinas, interior do Estado de São Paulo, no período de julho de 2007 a janeiro de 2008.

\section{MÉTODOS}

Neste estudo documental foram considerados 589 protocolos de recém-nascidos avaliados no programa de TANU, em um Hospital da rede pública do município de Campinas - SP, no período de julho de 2007 a janeiro de 2008, com a devida autorização do Diretor da Instituição. Esses protocolos de registro continham dados familiares, dados da gestação e do recém-nascido, antecedentes familiares, dados sobre indicadores de risco que podem estar presentes e resultados do exame de EOAET para as duas orelhas e do reflexo cócleo-palpebral (RCP).

Os protocolos analisados neste estudo registram as informações obtidas por uma fonoaudióloga por ocasião da TANU e, no caso de reavaliação, os resultados anotados no mesmo prontuário da instituição. Os indicadores de risco encontrados na amostra foram computados e os resultados lançados em planilha de dados no programa Excel 2003, onde foram elaborados gráficos, utilizando os recursos disponíveis.

Segundo as informações da instituição, o exame de EOAET foi realizado com o equipamento ILO 292 OAE - Otodynamics, nas frequências de 500 a $6000 \mathrm{~Hz}$, com intensidade de $70 \mathrm{~dB}$. O resultado foi registrado considerando as respostas das EOAET em relação sinal/ruído, tendo como critério: presente - respostas em três frequências ou mais e ausente - respostas em zero, uma ou duas frequências. Quando a avaliação no primeiro exame resultou em ausência de resposta, o recém-nascido foi encaminhado para um segundo exame. Aqueles que falharam também no segundo exame foram encaminhados para um terceiro exame e para avaliação otorrinolaringológica.

Este estudo foi avaliado e aprovado pelo Comitê de Ética em Pesquisa Protocolo 734/06.

\section{RESULTADOS}

Dos 589 protocolos analisados, 152 apresentam indicadores de risco $(25,8 \%)$. Os indicadores de risco observados na amostra encontram-se na Tabela 1. Nota-se que o histórico familiar de perda auditiva, isoladamente ou acompanhado de outros indicadores, predomina nesta amostra.

A Figura 1 evidencia que mais de $1 / 4$ dos protocolos apresentavam algum indicador de risco.

O número de indicadores de risco presentes em cada recém-nascido avaliado variou de 1 a 6 , como mostra a Figura 2.

A Figura 3 mostra o percentual dos neonatos com indicadores de risco que falharam no teste de EOAT e foram encaminhados a re-teste.

A Figura 4 evidencia que dos 36 neonatos da amostra encaminhados para re-teste, $6 \%$ não o concluíram e $19 \%$ não compareceram. 
Tabela 1 - Indicadores de risco presentes na amostra estudada $(n=152)$

\begin{tabular}{lcc}
\hline INDICADOR DE RISCO & FREQÜÊNCIA & $\%$ \\
\hline Histórico familiar de perda auditiva & 40 & 26,3 \\
Apgar de 0 a 4 no $1^{\circ}$ minuto & 36 & 23,6 \\
Medicamentos ototóxicos & 36 & 23,6 \\
Encubadora & 34 & 22,3 \\
Hiperbilirrubinemia & 33 & 21,7 \\
UTI Neonatal & 28 & 18,4 \\
Consanguinidade entre os pais & 12 & 7,8 \\
Peso inferior a 1500g & 11 & 7,2 \\
Asfixia perinatal & 4 & 2,6 \\
Infecção intra-uterina-sífilis & 3 & 1,9 \\
Ventilação mecânica & 2 & 1,3 \\
HIV materno & 2 & 1,3 \\
Apgar de 0 a 6 no 5o min & 2 & 1,3 \\
Incompatibilidade RH/ABO & 1 & 0,6 \\
Anomalias craniofaciais (fissura lábio-palatina) & 1 & 0,6 \\
Citomegalovírus & 1 & 0,6 \\
Infecção intra-uterina-toxoplasmose & 1 & 0,6 \\
\hline
\end{tabular}

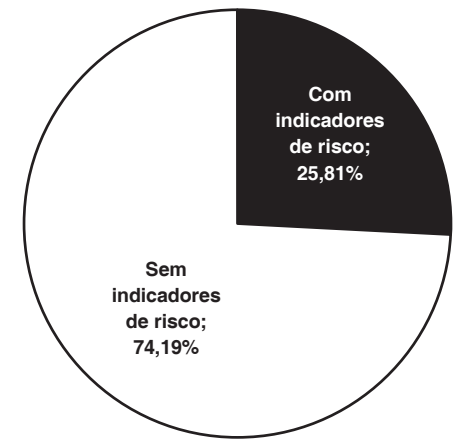

Figura 1 - Presença de indicadores na amostra $(n=589)$

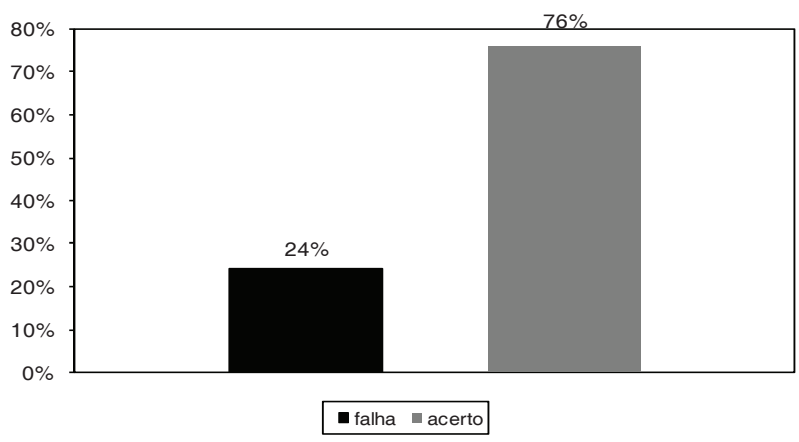

Figura 3 - Percentual de neonatos com indicadores de risco que falharam no primeiro exame $(n=152,24 \%)$

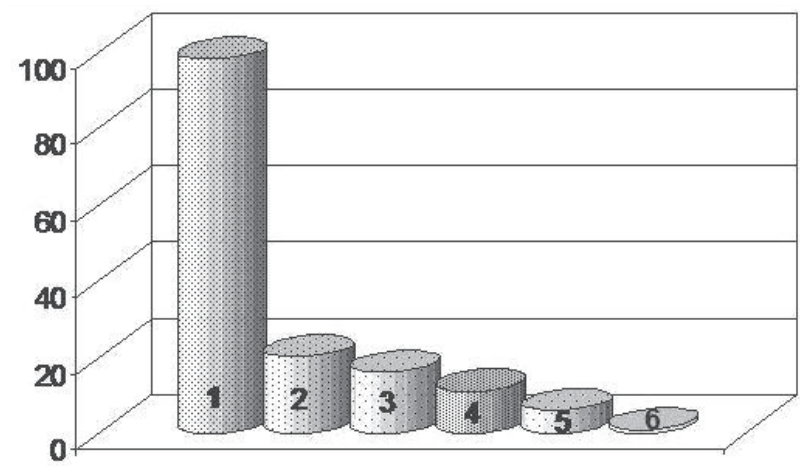

Figura 2 - Número de indicadores de risco presentes por neonato $(n=152)$

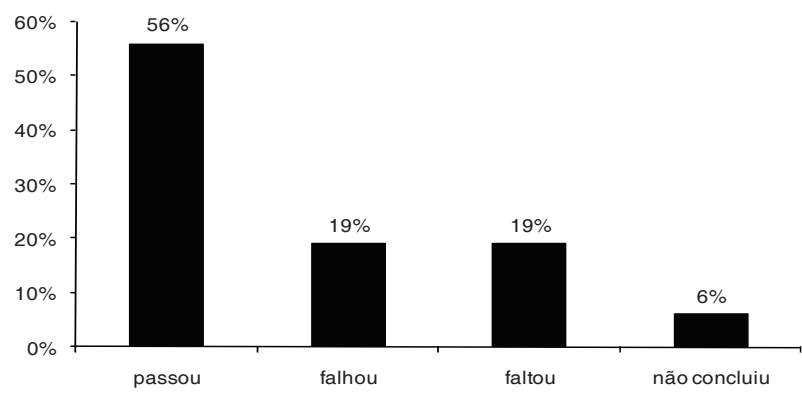

Figura 4 - Distribuição dos neonatos com indicadores de risco encaminhados, que falharam ou faltaram ou não concluíram o $2^{\circ}$ exame 


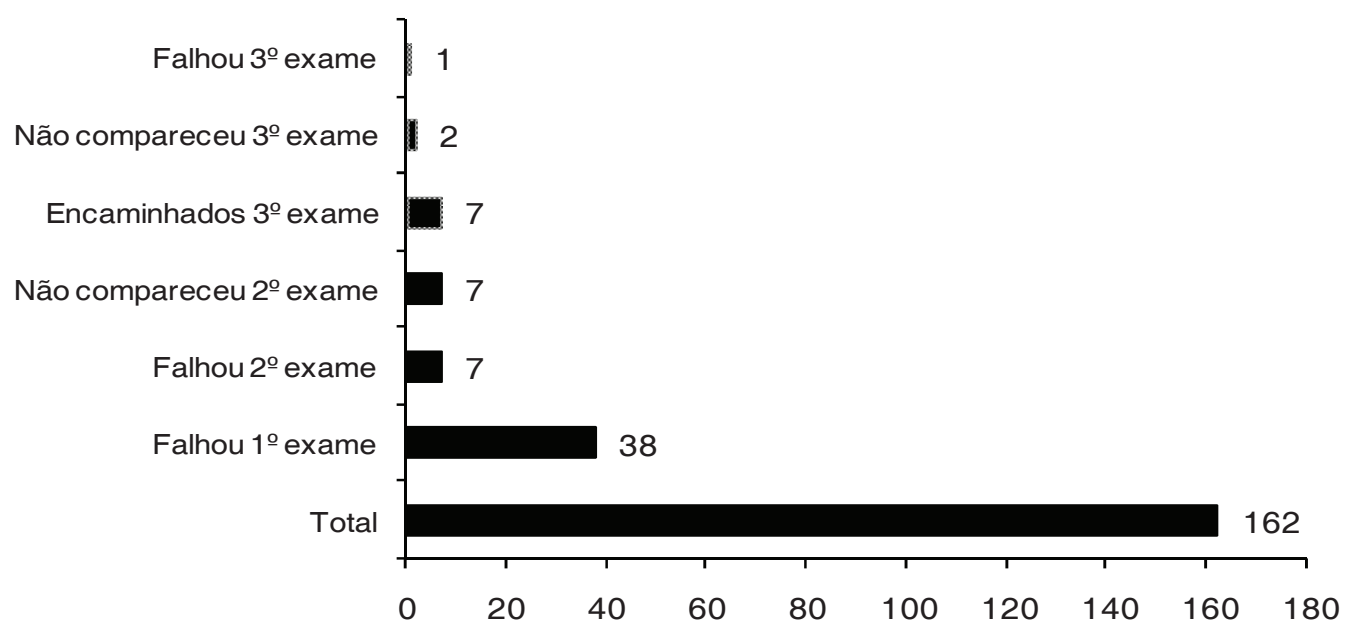

Figura 5 - Neonatos com indicadores de risco e resultados observados no protocolo de 3 etapas adotado na instituição

A Figura 5 mostra o filtro dos casos suspeitos realizado pela Triagem Auditiva Neonatal, no protocolo de três passos adotado pela Instituição.

\section{DISCUSSÃO}

De uma amostra de 589 protocolos de EOAET obtidos, $152(25,8 \%)$ apresentaram indicadores de risco compatíveis com aqueles definidos pelo Joint Comitee on Infant Hearing ${ }^{8}$.

Dos indicadores de risco observados na amostra, foram mais prevalentes: o histórico familiar de perda auditiva, o boletim Apgar de 0 a 4 no $1^{\circ}$ minuto, o uso de medicamentos ototóxicos, a permanência em incubadora, a hiperbilirrubinemia e a permanência em UTI Neonatal. Em 98 protocolos (aproximadamente $65 \%$ ) foi encontrado um único indicador de risco. Nos demais protocolos, 20 apresentavam dois indicadores; $16 \mathrm{com}$ três indicadores, $11 \mathrm{com}$ quatro indicadores, seis com cinco indicadores e um com seis indicadores. Esses resultados estão parcialmente de acordo com achados de outro estudo brasileiro ${ }^{23}$.

Dentre os componentes da amostra com indicadores de risco, 36 falharam no teste e foram encaminhados para o re-teste. Neste segundo exame, sete não compareceram e sete falharam, sendo encaminhados a um terceiro exame. Neste último, dois não compareceram e um falhou.

Do total das avaliações realizadas nessa amostra, apenas um neonato falhou nos três testes de EOAET. Este neonato apresentava três indicadores de risco: hiperbilirrubinemia, permanência em UTI e uso de medicamentos ototóxicos.
Esses dados indicam que o protocolo de três passos utilizado na maternidade estudada foi capaz de filtrar os casos de risco, sendo que dos 589 neonatos avaliados, apenas um manteve a falha nos três exames de EOAET. Esse protocolo de três passos evidenciou perda na continuidade do processo de nove recém-nascidos, o que representa $6 \%$ dos que apresentaram indicadores de risco $(n=152)$ e 1,5\% do total da amostra $(n=589)$. Esse índice de descontinuidade no processo está dentro do esperado por organizações nacionais ${ }^{7} \mathrm{e}$ internacionais ${ }^{8}$.

\section{CONCLUSÃO}

Este estudo permitiu identificar 152 protocolos com indicadores de risco em uma amostra de 589 neonatos de uma maternidade do interior do Estado de São Paulo. O número de indicadores de risco encontrado em cada neonato variou de um a seis. A maior parte deles apresentava um único indicador de risco. Considerando que a presença de indicadores de risco é importante para o monitoramento da saúde auditiva, pelo menos, até o terceiro ano de vida; como preconizado pelas organizações internacionais e nacionais, ressalta-se a importância das informações a esse respeito no protocolo de triagem auditiva neonatal universal e no encaminhamento para acompanhamento posterior dos neonatos, mesmo daqueles que tenham passado nos exames realizados na triagem auditiva.

\section{AGRADECIMENTOS}

À FAPESP (Bolsa de Iniciação Científica). 


\begin{abstract}
Purpose: to identify the hearing loss risk indicators in newborns at a public hospital facility in Campinas - São Paulo, in the period from July 2007 to January 2008. Methods: sample composed of 589 protocols of newborns evaluated in the Program of Universal Hearing Screening during the considered period. Results: 152 protocols (25.8\%) showed hearing loss risk indicators. Among them, the most prevalent were, in decreasing order: family antecedents (26.3\%); Apgar index from 0 to 4 in the first minute $(23.6 \%)$; ototoxic drug use $(23.6 \%)$, incubator permanence $(22.3 \%)$, hyperbilirubinemia of the newborn (21.7\%) and permanence in Neonatal Intensive Care Unit (18.4\%). Conclusion: the number of risk indicators found per newborn ranged from 1 to 6 . Among the newborns with hearing loss risk indicators, just one baby failed in the three evaluations conducted in the hearing screening program. This baby's protocol showed three hearing loss risk indicators.
\end{abstract}

KEYWORDS: Audiology; Risk Factors; Screening

\section{REFERÊNCIAS}

1. Magni C, Baus SC. Bases genéticas da deficiência auditiva. Rev Soc Bras Fonoaudiol. 2005; 10:239-44.

2. Uchôa NT, Procianoy RS, Lavinsky L, Sleifer P. Prevalência de perda auditiva em recém-nascidos de muito baixo peso. J Pediatria. 2003; 79(2):123-8.

3. Parving A, Hauch AM. The causes of profound hearing impairment in a school for the deaf: a longitudinal study. Br J Audiol. 1994; 28(2):63-9.

4. Morton NE. Genetic epidemiology of hearing impairment. Ann N Y Acad Sci. 1991; 630:16-31.

5. Souza ASN, Remião NML, Costa D, Linden A. Fatores de risco perinatal na gênese da surdez. Incidência e prevenção. [periódico on line] Rev Bras Otorrinolaringol. 1993; 59(2):129-31.

6. Yoshinaga-Itano C, Apuzzo ML. Identification of hearing loss after age 18 months is not early enough. Am Ann Deaf. 1998; 143(5):380-7.

7. Comitê Brasileiro sobre Perdas Auditivas na Infância. Resolução 01/99 [periódico on line] Disponível em: <http://www.unifesp.br/dotorrino/orl/ graduacao/roteiros/surdez_inf.doc>. Acesso em 23 mar de 2007.

8. American Academy of Pediatrics, Joint Committe on Infant Hearing. Year 2007 position statement: principles and guidelines for early hearing detection and intervention programs. Pediatrics. 2007; 120(4):898-921.

9. Ribeiro GR, Mitre El. Avaliação do conhecimento sobre triagem auditiva neonatal de pacientes no pós-parto imediato. Rev CEFAC. 2004; 6(3):294-9.

10. Pudo FA, Cicca FP, Ferreira VJA. Triagem auditiva comportamental neonatal. Rev CEFAC. 2002; 4(1):77-9.

11. Pádua FGM, Marone S, Bento RF, Carvallo RMM, Durante AS, Soares JC, et al. Triagem auditiva neonatal: um desafio para sua implantação. [periódico on line] Arq Int Otorrinolaringol. 2005; 9(3):189-94.

12. Manfredi AKS, Santos CB. Intervenção fonoaudiológica junto às mães ou responsáveis por recém-nascidos de risco para deficiência auditiva, precocemente detectada. Medicina. 2002; 35:70-7.

13. Azevedo MF. Emissões otoacústicas. In: Figueiredo MS. Conhecimentos essenciais para entender bem EOA e BERA. Coleção CEFAC. São José dos Campos: Pulso; 2003. p. 35-70.

14. Sociedade Brasileira de Pediatra. Comitê Brasileiro Sobre Perdas Auditivas na Infância [serial on the internet] Disponível em: URL: <http://www. sbp.com.br/show_item.cfm?id_categoria $=24 \& i d$ detalhe=520\&tipo=S>. Acesso em 19 set de 2007.

15. Vieira EP, Miranda EC, Azevedo MF, Garcia MV. Ocorrência dos indicadores de risco para a deficiência auditiva infantil no decorrer de quatro anos em um programa de triagem auditiva neonatal de um hospital público. Rev Bras Fonoaudiol. 2007; 12(3):214-20.

16. Kalatzis V, Petit C. The fundamental and medical impacts of recent progress in research on hereditary hearing loss. Hum Mol Genet. 1998; 7(10):1589-97.

17. Carraquillo MM, Zlotogora J, Barges S, Chakravarti A. Two different connexin 26 mutations in an inbred kindred segregation non-syndromic recessive deafness: implications for genetic studies in isolated populations. Hum Mol Genet. 1997; 6(12): 2163-72.

18. Kemp DT. Stimulate acoustic emissions from within the human auditory system. J Acoust Soc Am. 1978; 64(5):1386-91.

19. Lopes Filho O, Carlos RC. Emissões otoacústicas. In: Lopes Filho O, Rosimeire CC. 
Tratado de fonoaudiologia. São Paulo: Rocca; 1997. p. 221-37.

20. Ribeiro GG. Estudo da amplitude de resposta das emissões otoacústicas evocadas transientes em recém-nascidos a termo. Rev CEFAC. 2003; 5(2):165-7.

21. Munhoz MS, Ganança MM, Caovilla HH, Silva MLG. Otoemissões acústicas. In: Audiologia clínica. São Paulo: Atheneu; 2000. p. 121-48.

22. Isaac ML, Manfredi AKS. Diagnóstico precoce da surdezna infância. In: Simpósio: Implicações Clínicas

e Possibilidades Terapêuticas. Anais Eletrônicos.
Ribeirão Preto: USP, 2005; 2. Disponível em: <http://www.fmrp.usp.br/revista/2005/vol38n3e4/ 2a_diagnostico_precoce_surdez_na_infancia.pdf $>$. Acesso em: 29 out. 2007.

23. Tiensoli LO, Goulart LMHF, Resende LM, Colosimo EA. Triagem auditiva em hospital público de Belo Horizonte, Minas Gerais, Brasil: deficiência auditiva e seus fatores de risco em neonatos e lactentes. Cad Saúde Públ. 2007; 23(6):1431-41. 24. Gatto $\mathrm{Cl}$, Tochetto TM. Deficiência auditiva infantil: implicações e soluções. Rev CEFAC. 2007; 9(1):110-5.

DOI: 101590 / S1516 - 18462009005000020

RECEBIDO EM: 26/05/2008

ACEITO EM: 28/9/2008

Endereço para correspondência:

Elizabeth Oliveira Crepaldi de Almeida

Av. Maria Martins Otobonni, 210

Jacareí - SP

CEP: 12306-700

E-mail: nelson_almeida@uol.com.br 